Published in final edited form as:

Neurol Sci. 2017 November ; 38(11): 1977-1984. doi:10.1007/s10072-017-3069-4.

\title{
Fixational eye movements in Tourette syndrome
}

\author{
Aasef G. Shaikh $\# 1,2,3$, Shlomit Ritz Finkelstein $\# 4$, Ronald Schuchard ${ }^{5}$, Glen Ross ${ }^{6}$, and \\ Jorge L. Juncos ${ }^{7}$ \\ ${ }^{1}$ Depatment of Neurology, Case Western Reserve University, Cleveland, OH, USA \\ ${ }^{2}$ Daroff-DelOsso Ocular Motility Laboratory and Neurology Service, Louis Stokes VA Medical \\ Center, Cleveland, OH, USA \\ ${ }^{3}$ Department of Neurology, University Hospitals Case Medical Center, 11100 Euclid Avenue, \\ Cleveland, $\mathrm{OH} 44110$, USA \\ ${ }^{4}$ Department of Psychology, Emory University, Atlanta, GA, USA \\ ${ }^{5}$ Department of Neurosurgery, Stanford University, Stanford, CA, USA \\ ${ }^{6}$ VA Medical Center, Atlanta, GA, USA \\ ${ }^{7}$ Depatment of Neurology, Emory University, Atlanta, GA, USA \\ \# These authors contributed equally to this work.
}

\begin{abstract}
Studies of saccadic eye movements in subjects with Tourette syndrome (TS) have provided additional evidence that there is a link between TS symptoms and deficits in fronto-striatothalamic networks. These studies revealed impaired timing and inhibition of saccades. We compared fixational eye movements, such as microsaccades and ocular drifts, in subjects with TS and healthy controls.We measured horizontal and vertical eye positions with video-oculography in 14 subjects with Tourette syndrome. We found reduced microsaccade amplitude but increased time between adjacent microsaccades (intersaccadic interval). Hence, the rate of microsaccades was reduced in subjects with TS compared to controls. Measure of ocular stability during intersaccadic intervals revealed increased drift velocity and increased variance in eye position. We hypothesize that increased activity of the direct fronto-striatal pathway and the resulting reduction in basal ganglia outflow targeting the superior colliculus fixation zone affect the rate and amplitudeS of microsaccades in subjects with TS. The resulting impairment in frontal eye field fixation leads to increased drifts during intersaccadic interval in subjects with TS. Possible clinical implication for these results is that fixational eye movements can be objective biological markers of TS.
\end{abstract}

\section{Keywords}

Microsaccades; Gaze holding; Vision; Tics; Basal ganglia

Compliance with ethical standards: The experiment protocol of this study complied with the tenets of the Declaration of Helsinki and was approved by the Emory University Institutional Review Board. 


\section{Introduction}

Tourette syndrome (TS) is a neuropsychiatric disorder featuring motor and phonic tics [1] and significant comorbidities in about $50 \%$ of those affected. Converging lines of evidence suggest that there is imbalance in the activity of frontostriatal circuits resulting in enhanced transmission through the direct pathway [1]. Studies of eye movements in TS have provided an opportunity to delineate the underlying patho-physiology of its motor manifestations, especially the putative imbalance between the outflow of the direct and indirect pathways leading to tics and other behaviors in TS. Previous studies have described abnormalities in saccadic eye movements in TS [2-9]. The impairments in saccades in TS were as follows: (1) abnormal reaction time of reflexive saccades in prosaccades towards a novel target, as well as during anti-saccadic eye movement with subjects trying to inhibit reflexive saccade in favor of a voluntary mirror movement away from the target [2-6]. (2) Increased directional error during anti-saccade paradigm $[3,6,8]$. (3) Timing error in inhibiting the delayed-saccade task when the eye movements were made prematurely, that is, prior to the disappearance of the fixation point [5]. In sum, saccade abnormalities in TS suggest abnormal response inhibition and/or delayed initiation. Both of these are consistent with disruptions in fronto-striato-thalamic networks.

Fixational eye movements such as slow changes in the eye position, called drifts, and rapid re-fixations, called microsaccades, collectively reorganize the foveal coordinates of the environmental image to prevent neural adaptation and visual fading [10-14]. Thus during fixation, microsaccades serve a function analogous to that of visually guided saccades during visual exploration [15]. Both saccades and microsaccades are binocular, mostly conjugate, and both classes of eye movements follow stereotypical amplitude-velocity relationship [16]. Generation of saccades and microsaccades utilizes the same neural substrate $[17,18]$.

A number of factors modulate microsaccades. In normal population, their amplitude increases in darkness $[19,20]$ and their rate increases during tasks requiring high acuity [21]. Task difficulty [22], mental fatigue [23], fixation target size [24], and informativeness of the natural scene [13] are known to affect microsaccades. Mental fatigue and hypoxia also influence drift velocity [23, 25]. In hypokinetic rigid syndromes like parkinsonism, microsaccade amplitude is increased [26, 27]. TS is a disorder with "dopamine hyperexpression" in the fronto-striatal-thalamic pathways. We therefore predicted that fixational eye movements would be affected in subjects with TS.

\section{Methods}

The experiment protocol of this study complied with the tenets of the Declaration of Helsinki and was approved by the Emory University Institutional Review Board. All subjects provided written informed consent.

\section{Participants}

We compared ocular motor functions in 14 TS subjects ( 6 women and 8 men) aged 19 to 77 years (mean \pm standard deviation: $39.87 \pm 18.05$ ), and 15 healthy adults (10 women and 5 
men), aged 27-70 years (mean \pm standard deviation: $47.0 \pm 16.67$ ), with no personal or family history of tics or neuropsychiatric disorders. We excluded TS subjects who had motor tic affecting their neck. TS subjects were a cross section of tic severity with all having at least one comorbid condition, mainly attention deficit hyperactivity disorder (ADHD), generalized anxiety disorder with or without obsessive-compulsive disorder (OCD). Diagnosis of TS and its comorbidities was made using DSM-IV criteria by a movement disorders specialist who had followed each patient for at least 3 years. Table 1 depicts the patient profiles. Symptom severity was measured using standardized Yale Global Tic Severity Scale (YGTSS)—rating scales for tics [28], Yale-Brown Obsessive Compulsive Scale (Y-BOCS) - OCD [29], and Brown Symptom Inventory—rating scale for ADHD [30]. At the time of testing, the subjects who were on any pharmacotherapy had been on stable doses of medication for at least a month. They were also free of drug-induced side effects such as daytime sedation, dizziness, or gastrointestinal discomfort. The medications taken by each TS subject are listed in Table 1 . The subjects did not have any abnormality affecting pupillary sphincters, corneal surgery, or surgical pupil. Controls were on no psychotropic medication.

\section{Equipment, setting, and eye-movement recording}

We used high-resolution video-based eye tracker (D6 Remote Desktop Eye Tracking Optics, Applied Science Laboratories (ASL), Billerica, MA) to measure horizontal and vertical eye positions. The eye tracker uses the corneal reflection (the first Purkinje image) and the center of the pupil to measure the coordinates of the gaze position. Such technology therefore does not rely on pupil size which may change with light reflex or cognitive processes including thoughts and behaviors. The parameter that might affect detection of the center of the pupil is the irregular shape (for example post-surgical pupil), but none of our subjects had eye surgery or irregular pupils. We measured the gaze position in space-fixed coordinates, hence not registering eye movements due to the vestibulo-ocular reflex. Additional precautions were also taken to prevent any head movements. The subject sat on a high-back chair that was set in a fixed, stationary, position. The subject's head was supported by the back of the seat and was secured to the headrest using a cloth head restraint to prevent movements. TS, by nature of its pathology, is associated with verbal tic. We discarded the trials that were associated with any verbal tics, since the latter can lead to head motion (and VOR). The subjects' nasion was $65 \mathrm{~cm}$ away from the projection screen. The screen was $40.64 \mathrm{~cm}$ (1280 pixels) long and $30.48 \mathrm{~cm}$ (1024 pixels) high. Its background color was blue (0,0,126 in RGB). The fixation cross was positioned at the center of the screen and was $0.75^{\circ}$ in diameter. The horizontal and vertical eye positions were measured as the subject attempted to fixate the gaze on the visual target or on the fixation cross. We performed four trials in two sets. The data was sampled at $120 \mathrm{~Hz}$ temporal and 5 minarc spatial resolution; the visual range was $50^{\circ}$ horizontally and $40^{\circ}$ vertically. The entire experiment took up to 15 min including the break time. The digital output from the video-oculography machine and the distance between the projection screen and the subject's position were used to compute the visual angle (eye position) offline.

The eye position data were used for post hoc analysis. All eye position epochs were smoothened with Savitzky-Golay filter (polynomial order: 3; frame length: 11). The eye 
position was differentiated and smoothed with a Savitzky-Golay filter (polynomial order: 3; frame length: 21) to compute the eye velocity. The microsaccades were identified with Engbert algorithm [17]. We used composite vector of horizontal and vertical components for further analysis. Previous studies have shown that microsaccade amplitudes reach asymptote at $1^{\circ}[11,17]$, but we analyzed fixational saccades with all amplitudes since they may contribute to the overall fixational instability in TS. In order to exclude visually guided or voluntary saccade, we used saccades that did not accompany target shifts and were smaller than $4^{\circ}$ amplitude. The variables of interest were as follows: amplitudes, intersaccadic invervals, peak velocities, and drift velocities and variance in eye position during intersaccadic interval; data analysis was performed using custom-prepared software in Matlab $^{\mathrm{TM}}$ programming language [20]. Further analysis was performed in three steps. First, we examined, at the population level, whether there are differences in the amplitude or frequency (rate) of microsaccades. For this analysis, we compared the distributions of amplitude values obtained for each microsaccade and the inverse of intersaccadic interval (the direct surrogate of frequency). There are two possible ways to measure the rate of microsaccades; one is to find intersaccade interval and compute its inverse. The second way is to fit a sinusoid and get the value of frequency from the fitted sinusoid. The latter technique, however, was not applicable in our data that had no sinusoidal modulation of the position waveform. Hence, we utilized intersaccadic interval to mesure the rate of microsaccade. We implemented Kolmogorov-Smirnov test to investigate the differences amongst populations. In addition we also compared the median values and the range of distributions. Age may influence movement parameters. Therefore, we assessed whether the subjects' age influences the microsaccade amplitudes and frequencies. We then measured the differences in the regression fit of the amplitude and frequency with age, and assessed whether two regression fits are significantly different. Such analyses allowed us to determine whether microsaccades are different in patients with TS compared to healthy subjects. It also facilitated an investigation whether the differences are due to the subjects' age.

\section{Results}

\section{Eye movements during fixation and eye positions during intersaccadic intervals}

Figure 1a depicts an example of horizontal and vertical eye movements during 5-s epoch of steady fixation in a subject with TS, while Fig. 1b depicts the same phenomenon in a healthy subject. Two types of fixational eye movements were present in both examples. One type was rapid re-fixation featuring small saccades not visible to the naked eye. These fixational saccades or microsaccades were also associated with slow drifts in the eye position during intersaccadic intervals. We performed quantitative analysis of the amplitude, velocity, intersaccadic interval, and the regularity of the microsaccades and the variance in eye position during intersaccadic interval.

\section{Microsaccade amplitude}

Figure 1c illustrates the summary of the amplitudes of microsaccades measured in TS subjects and controls. We distributed microsaccades from each population amongst respective amplitude bins. We then normalized the proportion of microsaccades in a given bin to the size of the population. Such normalization allowed comparison of the distributions 
without being affected by uneven sample size [20]. Normalized occurrence of microsaccades is plotted on the $y$-axis while corresponding bin value is plotted on the $x$-axis. The normalized histogram compares the amplitude distribution in control subjects (blue lines) with that of the TS subjects (red line). The range of the amplitudes covering 66\% of the area under the curve in the healthy controls is $0.33-1.41^{\circ}$. The curve describing the TS population shows a leftward shift with the amplitude range comprising $66 \%$ of the area under the curve between $0.30-1.30^{\circ}$. These differences between the two curves are statistically significant (Kolmogorov- Smirnov statistics: $p=0.03$ ). The leftward shift of the curve depicting the TS population suggests more prevalent low- amplitude microsaccades in TS as compared to healthy subjects.

\section{Intersaccadic interval}

We measured the duration of intersaccadic interval quantifying the spacing of adjacent microsaccades. Figure 1d depicts a normalized histogram comparing the distribution of intersaccadic interval in subjects with TS and controls. The mean intersaccadic interval in TS was $1.40 \pm 1.30$ while in controls it was $0.90 \pm 0.70 \mathrm{~s}$. The range of intersaccadic interval was $0.19-1.88 \mathrm{~s}$ in healthy subjects and statistically different in TS subjects, $0.21-2.50 \mathrm{~s}$ (Kolmogorov-Smirnov statistics: $p<0.001$ ). Prolongation of intersaccadic interval suggests decreased rate of microsaccade generation, while increased range of the $66 \%$ area under the curve depicts increased irregularity in the occurrence of microsaccades in TS.

\section{Main-sequence analysis}

We measured the quantitative features of the saccade kinematics in the main-sequence where the amplitude of displacement is correlated to the corresponding peak velocity. Figure 1e depicts the summary of a main-sequence from TS subjects and control subjects. The scatter of the data points depicts a TS overlay over that of the controls. It is noteworthy that we utilized logarhthmic values of amplitude and velocity of saccades and microsaccades to accommodate the entire range of gaze shift in Fig. 1e. Smaller size of saccades, such as microsaccades, follow linear amplitude to velocity relationship, while larger size of saccades have non-linear relationship depicted by the Eq. 1 .

$$
\text { Peak saccade velocity }=\text { Constant value } 1 *\left(1-\mathrm{e}^{- \text {saccade amplitude/constant value } 2}\right)
$$

Logarithmic value of the saccade amplitude and velocity would, however, reveal linear relationship, regardless of their size. Hence, the comparison of the logarhithmic values, as depicted in Fig. 1e, allowed linear fit of all saccades (regardless of their size) using the same linear fit equation. The slope of the linear fit in TS was 0.60 and 0.62 in controls. The mainsequence suggests normal kinematic properties of microsaccades in TS.

\section{Fixation stability during intersaccadic intervals}

In order to measure fixation stability during intersaccadic intervals, we first measured the variance of eye positions. Figure $1 \mathrm{f}$ depicts the summary of the variances of eye positions in controls and in TS. The median variance of eye position in TS was $0.024^{\circ}$, and $95 \%$ 
confidence interval around the median of variance was in the range of $0.02-0.026^{\circ}$. In healthy subjects, the median variance was $0.02^{\circ}$ and $95 \%$ confidence interval around the median ranged between $0.017^{\circ}$ and $0.023^{\circ}$. The difference in the variance of eye position were small but reached statistical significance ( $p=0.01, t$ test).

\section{Drift velocity}

We measured the velocity of the eyes between adjacent microsaccades during drifts. Fig. $1 \mathrm{~g}$ illustrates the distribution of drift velocity in all the TS (red line) and the healthy subjects (blue line) in a normalized histogram. There was a shift in the peak of the curve from $1.79 \%$ in healthy subjects to $2.6 \%$ in TS suggesting significantly more instances of higher drift velocity in TS subjects than in healthy controls (Kolmogorov-Smirnov statistics: $p=$ 0.002). The range of drift velocities encompassing 66\% area under the curve was 1.60 $4.0^{\circ} \mathrm{s}$ in healthy subjects, the lower value of the range further shifted, but the range got narrower in TS $(1.90-3.60 \%)$.

\section{Lack of correlation with severity and comorbidities}

We qualitatively assessed the severity of TS and the associated comorbidities of ADHD and OCD using YGTSS, Brown ADD scale, and Y-BOCS, respectively. We found lack of correlation between the rating scale outcomes and the microsaccade amplitude, intersaccadic interval, and drift velocity. Table 2 depicts the coefficient of determination ( $r$-squared values) of each parameter of the fixational eye movements and the score obtained from the rating scales. We cannot comment on the potential effect of medications on these results other than pointing out that the medication types but not the doses of medications used by TS subjects were comparable across subjects.

Characteristics of microsaccades can vary with the age. In order to assess putative influence of the age on observed results, we measured whether regression fit correlating the frequency and amplitude with the age of the subject is significantly different in healthy subjects compared to TS. The slope and intercept of relationship of the amplitude with age was $-0.00005 \pm 0.003$ (fit $\pm 95 \%$ confidence interval) and $0.5 \pm 0.2$ in TS, while it was 0.01 \pm 0.003 and $0.05 \pm 0.17$ in healthy subjects. The slope and intercept of frequency relationship to the age was $0.04 \pm 0.01$ and $0.76 \pm 0.5$ in TS, and it was $0.001 \pm 0.01$ and 2.2 \pm 0.5 in healthy subjects. The difference in both comparisons was statistically significant ( $p$ $<0.01)$.

Effects of pharmacotherapy-We then asked whether pharamacotherapy used to treat symptoms of TS, even at therapeutic doses, affects the microsaccade amplitude and frequency. Therefore, we classified the subjects in TS group among those with pharmacotherapy and who were drug free. There was no difference in the amplitude (ANOVA, $p=0.14$ ) or frequency of microsaccades (ANOVA, $p=0.4$ ) between two groups.

\section{Discussion}

We found three key findings in fixational eye movements in subjects with TS with comorbid OCD and ADD: (i) The amplitude of microsaccades was relatively reduced, (ii) rate of 
microsaccade was reduced, and (iii) the ocular drifts had higher velocity. These results are consistent with known path-ophysiology in TS centered on abnormal function of frontostriatal circuits and altered basal ganglia output to the superior colliculus.

Basal ganglia is an important relay center for indirect projections from frontal and parietal eye fields to the superior colliculus [31]. The eye movement-related cortical neurons project to the caudate nucleus [31]. The caudate sends inhibitory direct projections to the substantia nigra pars reticulata and indirectly to the subthalamus via the external segment of the globus pallidus [32] (Fig. 2). Substantia nigra pars reticulata maintains tonic GABAergic inhibition on the superior colliculus [33] (Fig. 2). Transient cessation of these neurons, in preparation for the voluntary saccade, leads to timely saccade initiation [34, 35]. Disruption in the activity of this network is known to result in saccade abnormalities [36].

The studies of eye movements in subjects with TS have suggested difficulties in the timing of saccade execution and the inhibition of preprogrammed motor commands supporting the existence of abnormal fronto-striatal circuit abnormalities [2-9, 17]. Such impairments are also known to exist in motor deficits, such as tics, hence providing further insight into the pathophysiology of TS as a disorder of inhibition and timing [2-9, 17]. TS might result from imbalance in activity of direct and indirect pathway, but unlike parkinsonism the direct pathway is relatively overactive, therefore reducing the activity of substantia nigra pars reticulata and subsequent disinhibition of the superior colliculus (Fig. 2). Latter might change the threshold level of pre-saccadic activity required to initiate a saccade. Changes in threshold of pre-saccadic activity might affect the frequency and amplitudes of microsaccades as we noted in TS.

Our findings of reduced microsaccade amplitude are consistent with findings from other classes of saccades in TS [4, 5]. Our subjects also had increased drift velocity during intersaccadic interval. Such phenomenon could be attributed to altered function of the frontal eye field fixation neurons [35]. Previous studies investigated the ability of subjects with TS to smoothly track a moving target (pursuit) and their ability to hold gaze on a steady target. The results were conflicting: one investigation discovered increased incidence of intrusive saccades disrupting steady fixation [36], while a former investigation revealed normal fixation stability in subjects with TS [9]. The measure of fixation stability in the presence (or absence) of a distracting stimulus was an important caveat in the interpretation of the affirmative results [36]. Although incidence of intrusive saccades was increased, there was no quantification of their kinematics [36]. We asked whether TS featuring imbalance in indirect and direct basal ganglia pathways as well as impairments in fronto-striatal network affect fixational eye movements.

We found no correlation between microsaccade amplitude, intersaccadic interval, and drift velocity, and the age, clinical severity of TS, and its comorbidities of ADD and OCD. Hence, the fixation impairment in TS is not linearly determined by symptom severity or the subjects' age. It is also possible that increased drift velocity in subjects with TS was merely a reflection of associated ADD, where subjects could not attend to fixate on the target. Lack of attention, however, would also increase the amplitude and/or the frequency of microsaccades requiring larger or more frequent re-fixations. However, the microsaccade 
amplitude and rate were reduced in our TS patients. None of our subjects had TS without ADD or OCD; hence, we could not comment on fixation stability in isolated TS. We used first Purkinje image and the center of the pupil to track the coordinates of the gaze; hence, there was no influence of pupil size on our measures.

The finding of reduced microsaccade amplitude and frequency in TS differed from that in subjects with disorders of visual system such as amblyopia [37], refractive error [38], or degenerative disorders such as Parkinson's disease or progressive supranuclear palsy [26, 27]. Disorders of visual system such as amblyopia increases the amplitude of microsaccades and reduces its frequency; refractive error affects the amplitude of microsaccades [37, 38]; the degenerative disorder such as Parkinson's disase and progressive supranuclear palsy increases the amplitude of microsaccades and increases their frequency [26, 27].

To epitomize, in TS subjects with ADD and OCD we found abnormal fixational eye movements. The microsaccade amplitude was reduced and intersaccadic interval was increased. These deficits supported abnormal fronto-striatal activity and impairment in the basal ganglia output to the collicular fixation zone. Increased drift velocities in subjects with TS might suggest suboptimal function of frontal eye field fixation sensitive neurons. There is paucity of objective therapeutic markers for motor disorders in TS; studies of gaze holding and microsaccades can be a sensitive outcome parameter.

\section{Acknowledgments}

Funding statement: AS is supported by grant from Dystonia Medical Research Foundation and Dystonia Coalition. The authors have no financial conflicts related to the contents included in this manuscript.

\section{References}

1. Singer HS (1997) Neurobiology of Tourette syndrome. Neurol Clin 15(2):357-379 [PubMed: 9115467]

2. Straube A, Mennicken JB, Riedel M, Eggert T, Muller N (1997) Saccades in Gilles de la Tourette's syndrome. Mov Disord 12(4): 536-546 [PubMed: 9251072]

3. Farber RH, Swerdlow NR, Clementz BA (1999) Saccadic performance characteristics and the behavioural neurology of Tourette's syndrome. J Neurol Neurosurg Psychiatry 66(3):305-312 [PubMed: 10084528]

4. Munoz DP, Le Vasseur AL, Flanagan JR (2002) Control of volitional and reflexive saccades in Tourette's syndrome. Prog Brain Res 140:467-481 [PubMed: 12508609]

5. LeVasseur AL, Flanagan JR, Riopelle RJ, Munoz DP (2001) Control of volitional and reflexive saccades in Tourette's syndrome. Brain 124(Pt 10):2045-2058 [PubMed: 11571221]

6. Mostofsky SH, Lasker AG, Singer HS, Denckla MB, Zee DS (2001) Oculomotor abnormalities in boys with tourette syndrome with and without ADHD. J Am Acad Child Adolesc Psychiatry 40(12):1464-1472 [PubMed: 11765293]

7. Nomura Y, Fukuda H, Terao Y, Hikosaka O, Segawa M (2003) Abnormalities of voluntary saccades in Gilles de la Tourette's syndrome: pathophysiological consideration. Brain and Development 25(Suppl 1):S48-S54 [PubMed: 14980373]

8. Narita AS, Shawkat FS, Lask B, Taylor DS, Harris CM (1997) Eye movement abnormalities in a case of Tourette syndrome. Dev Med Child Neurol 39(4):270-273 [PubMed: 9183269]

9. Bollen EL, Roos RA, Cohen AP, Minderaa RB, Reulen JP, Van de Wetering BJ, Van Woerkom TC, Buruma OJ (1988) Oculomotor control in Gilles de la Tourette syndrome. J Neurol Neurosurg Psychiatry 51(8):1081-1083 [PubMed: 3216209] 
10. Martinez-Conde S, Macknik SL, Troncoso XG, Dyar TA (2006) Microsaccades counteract visual fading during fixation. Neuron 49(2):297-305 [PubMed: 16423702]

11. Martinez-Conde S, Macknik SL, Troncoso XG, Hubel DH (2009) Microsaccades: a neurophysiological analysis. Trends Neurosci 32(9):463-475 [PubMed: 19716186]

12. Costela FM, McCamy MB, Macknik SL, Otero-Millan J, Martinez-Conde S (2013) Microsaccades restore the visibility of minute fo-veal targets. PeerJ 1:e119 [PubMed: 23940832]

13. McCamy MB, Macknik SL, Martinez-Conde S (2014) Different fixational eye movements mediate the prevention and the reversal of visual fading. J Physiol 592(19):4381-4394 [PubMed: 25128571]

14. McCamy MB, Otero-Millan J, Macknik SL, Yang Y, Troncoso XG, Baer SM, Crook SM, Martinez-Conde S (2012) Microsaccadic efficacy and contribution to foveal and peripheral vision. J Neurosci 32(27):9194-9204 [PubMed: 22764228]

15. Otero-Millan J, Macknik SL, Langston RE, Martinez-Conde S (2013) An oculomotor continuum from exploration to fixation. Proc Natl Acad Sci U S A 110(15):6175-6180 [PubMed: 23533278]

16. Otero-Millan J, Troncoso XG, Macknik SL, Serrano-Pedraza I, Martinez-Conde S, Saccades and microsaccades during visual fixation, exploration, and search: foundations for a common saccadic generator, J Vis 8(14) (2008) 21 1-18

17. Rolfs M, Kliegl R, Engbert R, Toward a model of microsaccade generation: the case of microsaccadic inhibition, J Vis 8(11) (2008) 5 1-23

18. Hafed ZM, Goffart L, Krauzlis RJ (2009) A neural mechanism for microsaccade generation in the primate superior colliculus. Science 323(5916):940-943 [PubMed: 19213919]

19. Young LR, Sheena D (1975) Eye-movement measurement techniques. Am Psychol 30(3):315-330 [PubMed: 1137223]

20. Ghasia FF, Shaikh AG (2015) Experimental tests of hypotheses for microsaccade generation. Exp Brain Res 233(4):1089-1095 [PubMed: 25563497]

21. Ko HK, Poletti M, Rucci M (2010) Microsaccades precisely relocate gaze in a high visual acuity task. Nat Neurosci 13(12):1549-1553 [PubMed: 21037583]

22. Siegenthaler E, Costela FM, McCamy MB, Di Stasi LL, Otero-Millan J, Sonderegger A, Groner R, Macknik S, Martinez-Conde S (2014) Task difficulty in mental arithmetic affects microsaccadic rates and magnitudes. Eur J Neurosci 39(2):287-294 [PubMed: 24438491]

23. Di Stasi LL, McCamy MB, Catena A, Macknik SL, Canas JJ, Martinez-Conde S (2013) Microsaccade and drift dynamics reflect mental fatigue. Eur J Neurosci 38(3):2389-2398 [PubMed: 23675850]

24. McCamy MB, Najafian Jazi A, Otero-Millan J, Macknik SL, Martinez-Conde S (2013) The effects of fixation target size and luminance on microsaccades and square-wave jerks. PeerJ 1:e9 [PubMed: 23638403]

25. Di Stasi LL, Cabestrero R, McCamy MB, Rios F, Catena A, Quiros P, Lopez JA, Saez C, Macknik SL, Martinez-Conde S (2014) Intersaccadic drift velocity is sensitive to short-term hypobaric hypoxia. Eur J Neurosci 39(8):1384-1390 [PubMed: 24877213]

26. Otero-Millan J, Serra A, Leigh RJ, Troncoso XG, Macknik SL, Martinez-Conde S (2011) Distinctive features of saccadic intrusions and microsaccades in progressive supranuclear palsy. J Neurosci 31(12):4379-4387 [PubMed: 21430139]

27. Otero-Millan J, Schneider R, Leigh RJ, Macknik SL, Martinez-Conde S (2013) Saccades during attempted fixation in parkinsonian disorders and recessive ataxia: from microsaccades to squarewave jerks. PLoS One 8(3):e58535 [PubMed: 23516502]

28. Leckman JF, Riddle MA, Hardin MT, Ort SI, Swartz KL, Stevenson J, Cohen DJ (1989) The Yale Global Tic Severity Scale: initial testing of a clinician-rated scale of tic severity. J Am Acad Child Adolesc Psychiatry 28(4):566-573 [PubMed: 2768151]

29. Goodman WK, Price LH, Rasmussen SA, Mazure C, Delgado P, Heninger GR, Charney DS, The Yale-Brown Obsessive Compulsive Scale. II. Validity, Arch Gen Psychiatry 46(11) (1989) 1012-6 [PubMed: 2510699]

30. Brown RT, Freeman WS, Perrin JM, Stein MT, Amler RW, Feldman HM, Pierce K, Wolraich ML (2001) Prevalence and assessment of attention-deficit/hyperactivity disorder in primary care settings. Pediatrics 107(3):E43 [PubMed: 11230624] 
31. Hikosaka O, Takikawa Y, Kawagoe R (2000) Role of the basal ganglia in the control of purposive saccadic eye movements. Physiol Rev 80(3):953-978 [PubMed: 10893428]

32. Nambu A, Tokuno H, Takada M (2002) Functional significance of the cortico-subthalamo-pallidal 'hyperdirect' pathway. Neurosci Res 43(2):111-117 [PubMed: 12067746]

33. Fisher RS, Buchwald NA, Hull CD, Levine MS (1986) The GABAergic striatonigral neurons of the cat: demonstration by double peroxidase labeling. Brain Res 398(1):148-156 [PubMed: 3542121]

34. Francois C, Percheron G, Yelnik J (1984) Localization of nigrostriatal, nigrothalamic and nigrotectal neurons in ventricular coordinates in macaques. Neuroscience 13(1):61-76 [PubMed: 6387531]

35. Wurtz RH, Hikosaka O (1986) Role of the basal ganglia in the initiation of saccadic eye movements. Prog Brain Res 64:175-190 [PubMed: 3523602]

36. Tajik-Parvinchi DJ, Sandor P (2011) Smooth pursuit and fixation ability in children with Tourette syndrome. Cogn Behav Neurol 24(4):174-186 [PubMed: 22134192]

37. Shaikh AG, Otero-Millan J, Kumar P, Ghasia FF (2016) Abnormal fixational eye movements in amblyopia. PLoS One 11(3):e0149953 [PubMed: 26930079]

38. Ghasia FF, Shaikh AG (2015) Uncorrected myopic refractive error increases microsaccade amplitude. Invest Ophthalmol Vis Sci 
A
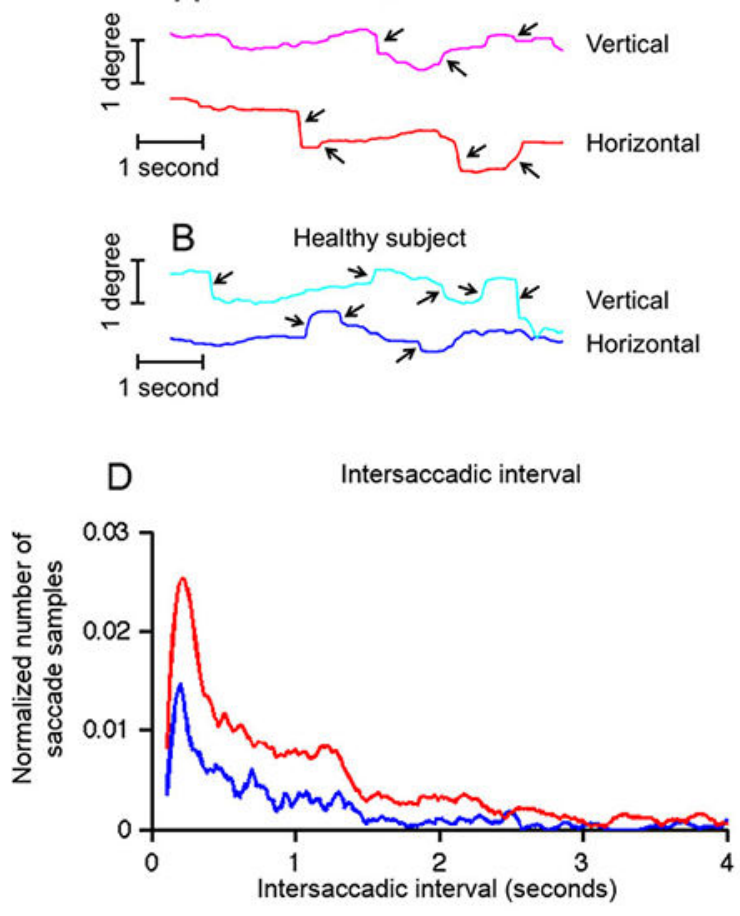

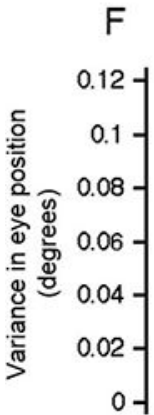

Variance of eye position during fixation

Fig. 1.
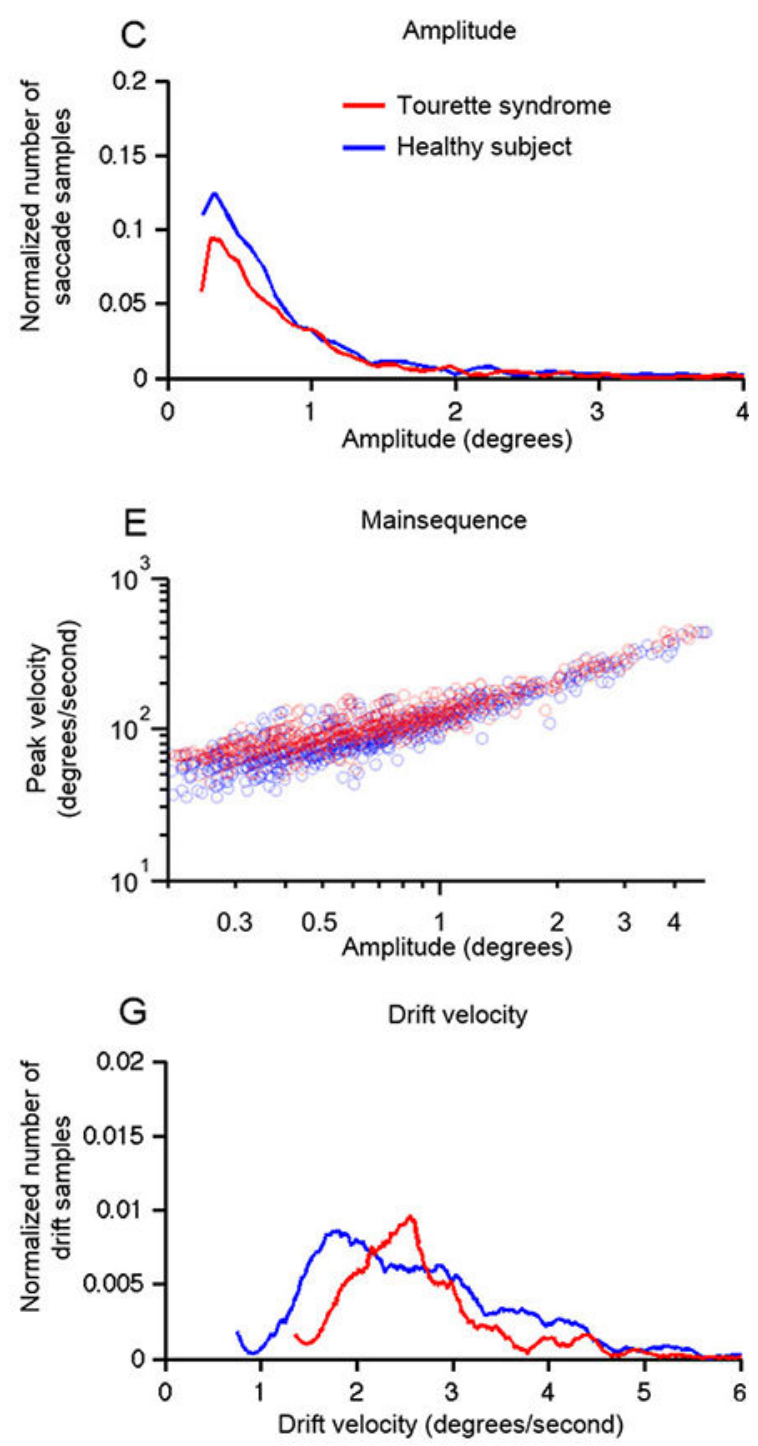

a An example of $5 \mathrm{~s}$ of gaze holding in a TS subject. Vertical eye positions are plotted in magenta color, while red lines are horizontal eye positions. b An example of $5 \mathrm{~s}$ of gaze holding in a healthy subject. Cyan lines are vertical eye positions, while blue lines are horizontal. In both panels, the vertical and the horizontal eye positions are plotted on the $y$ axis, while the corresponding time is on the $x$-axis. Each arrow illustrates a microsaccade. $\mathbf{c}$ The distribution of microsaccade amplitudes is depicted in normalized histogram. Event rate was normalized with the cumulative sum of the histogram to facilitate comparison between two uneven sample sizes. Amplitude bins are plotted on $X$-axis. Blue lines depict healthy controls while TS subjects are illustrated with red line. d Normalized distribution of intersaccadic intervals. Event rate, normalized with the cummulative sum, is plotted on $y$ axis, while $X$-axis depicts the bins of intersaccadic interval. e Main-sequence analysis comparing the peak velocity and the displacement amplitude of each microsaccade. Each symbol depicts one event. Blue symbols represent healthy subjects, while red symbols represent subjects with TS. f Variance of eye position during intersaccadic interval is plotted 
on $y$-axis. Each box plot depicts one group; blue is healthy subjects while TS subjects are summarized in red box plot. $\mathrm{g}$ Normalized distribution of drift velocity. The event rate, normalized with the cumulative sum of the histogram, is plotted on the $y$-axis while the drift velocity is plotted on the $x$-axis. Red lines depict Tourette syndrome, while blue lines represent healthy subjects 
Normal

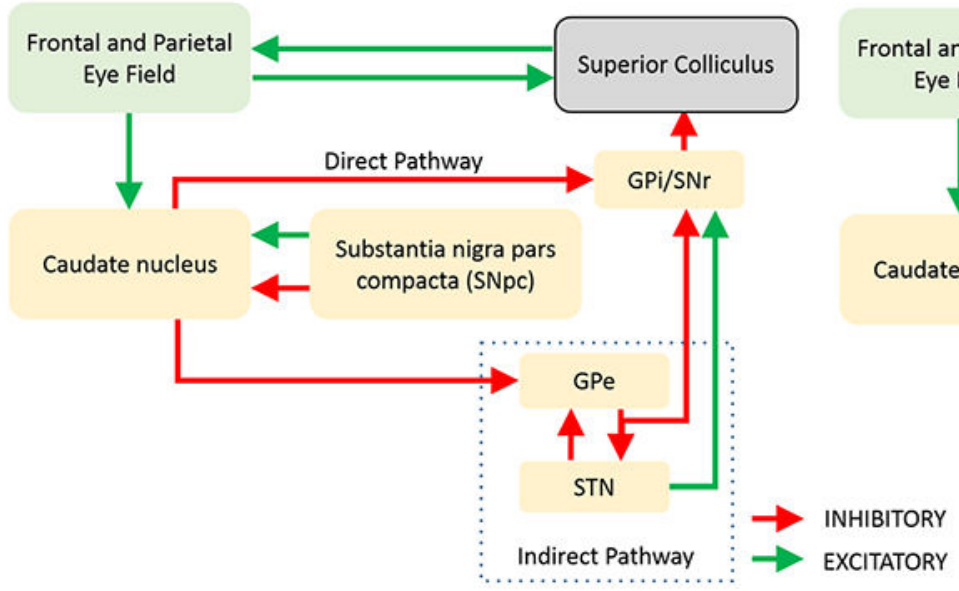

Tourette syndrome
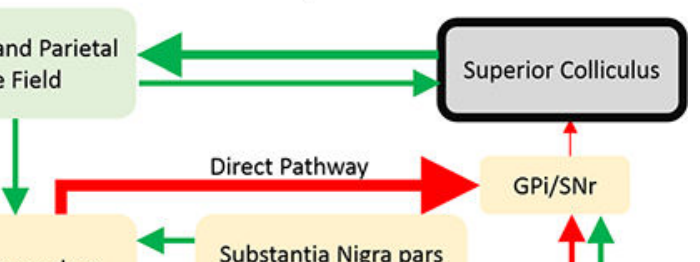

date nucleus

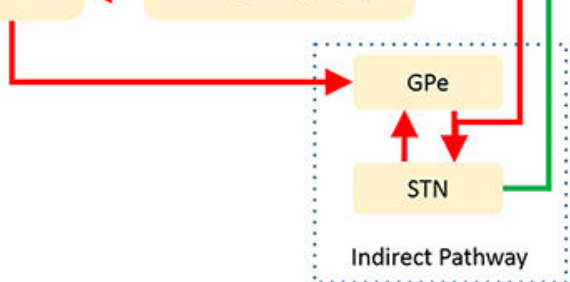

Fig. 2.

Schematic diagram depicting the normal network connections between the parietal and frontal cortex, striatum, pallidum, subthalamus, and the superior colliculus in programming of saccades. Red arrows depict inhibitory connections, while green arrows are excitatory. Thicker arrows in the panel depicting Tourette syndrome depicts overactivity, while thinner arrows illustrated reducted activity. Disinhibition of the superior colliculus is illustrated with thick border of the corresponding box 


\section{Table 1}

Patient profiles

\begin{tabular}{lllllll}
\hline ID & Gender & Age & TS (YGTSS) & OCD (Y-BOCS) & Brown ADD Scale & Medications \\
\hline 1 & F & 25 & 20 & $(2,2,2,2,2,2,2,2,2,2)$ & $(16,9,7)$ & Venlaflaxine \\
2 & M & 67 & 30 & $(4,2,2,4,4,2,1,2,2,2)$ & $(4,2,24)$ & None \\
3 & M & 27 & 35 & $(2,2,2,2,1,1,14,2,2)$ & $(11,13,6)$ & None \\
4 & M & 77 & & & & Aripriprazole, Cilatopram \\
5 & M & 39 & 30 & $(3,2,2,2,3,2,3,2,2,3)$ & $(7,8,16)$ & None \\
6 & F & 42 & 18 & $(1,0,1,1,2,0,0,0,0,0)$ & $(2,0,0)$ & Guanfancine \\
7 & F & 31 & & & & Aripriprazole, Clonazepam \\
8 & M & 45 & 20 & $(1,1,0,0,1,0,0,0,0,0)$ & $(9,7,10)$ & None \\
9 & M & 33 & 10 & $(1,0,0,1,1,0,0,0,0,0)$ & $(8,9,9)$ & Haloperidol \\
10 & F & 25 & & & & None \\
11 & F & 66 & & $(1,0,0,0,1,1,0,1,0,1)$ & $(2,0,0)$ & Buproprion \\
12 & M & 19 & 35 & $(2,1,3,2,1,2,1,2,3,2)$ & $(4,24,6)$ & Lisdexamfetamine \\
13 & F & 28 & 25 & $(2,2,1,1,2,2,1,1,1,1)$, & $(3,7,29)$ & None \\
14 & M & 30 & 30 & $(2,1,2,0,1,1,1,1,1,1)$ & $(10,11,13)$ & Fluphenazine, Phenargan \\
\hline
\end{tabular}

Neurol Sci. Author manuscript; available in PMC 2018 November 21. 


\section{Table 2}

Coefficient of determination ( $r$-squared values) of each parameter of the fixational eye movements and the score obtained from the rating scales

\begin{tabular}{lcll}
\hline Slope & & & \\
\hline & Drift & Intersaccadic interval & Amplitude \\
YGTSS & -0.03 & 0.01 & -0.003 \\
Y-BOCS & -0.06 & 0.004 & 0.0004 \\
ADD brown & -0.05 & 0.002 & -0.001 \\
Correlation coefficient & & \\
& Drift & Intersaccadic interval & Amplitude \\
YGTSS & 0.05 & 0.004 & 0.02 \\
Y-BOCS & 0.1 & 0.0007 & 0.0004 \\
ADD brown & 0.2 & 0.0002 & 0.01 \\
\hline
\end{tabular}

Neurol Sci. Author manuscript; available in PMC 2018 November 21. 